\title{
Hashimoto's encephalopathy in a patient with septal panniculitis: a case report
}

\author{
C. Cosso, M. Ghio, M. Cutolo \\ Clinica Reumatologica, Policlinico San Martino, Genova, Italy
}

\begin{abstract}
SUMMARY
Hashimoto's encephalopathy (HE) is an autoimmune form of encephalopathy, associated with autoimmune thyroiditis. Its prevalence is estimated to be 2:100,000. HE is characterized by behavioral changes, mental confusion, dysarthria, ataxia, psychosis, paranoia, convulsions, hallucinations, headache and hyperthermia. Elevated thyroid antibodies are necessary for diagnosis and the disease responds dramatically to glucocorticoid therapy. We describe a patient with HE and panniculitis, an association reported twice in the literature.
\end{abstract}

Key words: Hashimoto; panniculitis; encephalitis.

Reumatismo, 2018; 70 (4): 268-269

\section{INTRODUCTION}

$\mathrm{H}$ ashimoto's encephalopathy (HE) is a non-infectious, autoimmune form of encephalopathy, associated with autoimmune thyroiditis. Its prevalence is estimated to be $2: 100,000$ (1). HE is characterized by behavioral changes, mental confusion, dysarthria, ataxia, psychosis, paranoia, convulsions, hallucinations, headache and hyperthermia. Elevated thyroid antibodies are necessary for diagnosis. The disease responds dramatically to glucocorticoid therapy (1-5).

We describe a patient with HE and panniculitis, an association reported twice in the literature: a child with panniculitis, vitiligo and thyroid dysfunction (6) and a 57-year-old woman with panniculitis, primary biliary cirrhosis (PBC) and Hashimoto's thyroiditis (7).

\section{CASE REPORT}

A 70-year old man was admitted to our unit for panniculitis, fever (up to $38^{\circ} \mathrm{C}$ ) and polyarthralgia. His previous domiciliary treatment was beclometasone $2 \mathrm{mg}$ /day for 3 months. He was in therapy for thyroid dysfunction with L-thyroxine $50 \mathrm{mcg} /$ day. $\mathrm{TSH}$ value was $5.43 \mathrm{mIU} / \mathrm{L}$, while T4 free was $9.37 \mathrm{ng} / \mathrm{L}$. Electrolytes, creatinine, arterial blood gases and $\mathrm{pH}$ were in range. Glycemic level was $132 \mathrm{mg} / \mathrm{dL}$. His clinical history included recurrent orchitis and Hashimoto's thyroiditis. Anti-thyroglobulin antibodies $(805.1 \mathrm{U} / \mathrm{mL})$ and anti-thyroid peroxidase $(408 \mathrm{U} / \mathrm{mL})$ were elevated. Anti-neutrophil cytoplasmic antibodies, antinuclear antibodies, and the ACE test were negative. Complement fractions levels were in range. Intestinal function was regular; there was no abdominal pain or other symptoms suggesting inflammatory bowel disease and colonoscopy showed no endoscopic or histologic alterations. There was no lymphadenopathy at chest CT compatible with sarcoidosis. During physical examination, the patient was awake, oriented and collaborating. He presented several hypodermic nodules in the four limbs. Histology of bioptic specimens confirmed septal panniculitis on the basis of the presence of large epidermal septa and sparse inflammatory infiltrates involving subcutaneous fat with activated lymphocytes in active lesions. Older lesions were characterized by fibrosis of the widened septa with sparse infiltrates of histiocytes, perivascular lymphocytes and fibrin deposits. A biopsy of the intestinal mucosa was negative. A positron-emission tomography (PET) showed 
diffuse uptake of the thyroid gland (SUV 7.5). Ultrasonography showed multinodular goiter which was hypervascularized at power Doppler. Nothing emerged from total-body CT scanning.

After tapering beclometasone to $0.5 \mathrm{mg} /$ day in 15 days, the patient presented sudden alteration of consciousness, with hyperpyrexia up to $39.5^{\circ} \mathrm{C}$, headache, psychosis, ataxia, dysphonia, drowsiness and mental confusion. Nuchal rigidity or lesions at contrast-enhanced CT brain scan were absent. C-reactive protein level (3.4 to $532 \mathrm{mg} / \mathrm{L})$ and leukocytes number $(4.32$ to $\left.9.86 \times 10^{9} / \mathrm{L}\right)$ increased. The electroencephalogram demonstrated lazy background activities. MRI was not performed, neither was a lumbar puncture in consideration of the serious deterioration of the general condition and state of consciousness of the patient. In the hypothesis of $\mathrm{HE}$, treatment with dexamethasone ev 16 mg was immediately started, in accordance with accepted diagnostic criteria (7) (Table I). No symptomatic treatment for his psychiatric manifestations was started. An immediate and permanent improvement was seen, with the disappearance of psychotic symptoms. Glucocorticoids were tapered to $12.5 \mathrm{mg}$ of prednisone/day in 4 weeks, with improvement of panniculitis and disappearance of arthralgia. As maintenance treatment, prednisone was tapered to 7.5 $\mathrm{mg} / \mathrm{die}$ in association with cyclosporin A $150 \mathrm{mg} / \mathrm{die}$. In the following 24 months, no other acute exacerbation occurred and no neurologic sequelae remained.

\section{DISCUSSION AND CONCLUSIONS}

$\mathrm{HE}$ is an autoimmune disease that causes cerebral vasculitis and directed injury against common brain-thyroid antigens. This hypothesis is supported by the prevalence in female population, by the association with other autoimmune diseases and by brain biopsy that shows mild lymphocytic infiltration of small vessels $(8,9)$. Thyroid functionality should be checked in those patients with encephalitis of unknown etiology and HE should be sup-
Table I - Diagnostic criteria for Hashimoto's encephalopathy.

Clinical presentations

Encephalopathy with cognitive impairment

Encephalopathy with psychiatric manifestation

Encephalopathy with partial or generalized seizures

Encephalopathy with focal neurological deficits or alteration of consciousness

Laboratory test

Presence of high titer anti-TPO

Exclusion of neurological disease

Exclusion of neurological infection, toxic, and metabolic disorder

Response to treatment

Patient's neurological status return to baseline level after steroids therapy

posed especially in presence of other autoimmune disease, such as panniculitis, or in those patients with thyroid dysfunction and neurological signs occurred while tapering the corticosteroids.

\section{REFERENCES}

1. Sadan O, Seyman E, Ash EL, et al. Adult-onset temporal lobe epilepsy, cognitive decline, multi-antiepileptic drug hypersensitivity, and Hashimoto's encephalopathy: two case studies. Epilepsy Behav Case Rep. 2013; 1: 132-5.

2. Olmez I, Moses H, Sriram S, et al. Diagnostic and therapeutic aspects of Hashimoto's encephalopathy. J Neurol Sci. 2013; 331: 67-71.

3. Mahmud FH, Lteif AN, Renaud DL, et al. Steroid-responsive encephalopathy associated with Hashimoto's thyroiditis in an adolescent with chronic hallucinations and depression: case report and review. Pediatrics. 2003; 112: 686-90.

4. Kothbauer-Margreiter I, Sturzenegger M, Komor J, et al. Encephalopathy associated with Hashimoto thyroiditis: diagnosis and treatment. J Neurol. 1996; 243: 585-93.

5. Payer J, Petrovic T, Lisy L, et al. Hashimoto encephalopathy: a rare intricate syndrome. Int J Endocrinol Metab. 2012; 10: 506-14.

6. Mirza B, Muir J, Peake J, et al. Connective tissue panniculitis in a child with vitiligo and Hashimoto's thyroiditis. Australas J Dermatol. 2006: 49-52.

7. Herr W, Lohse AW, Spahn TW, et al. Nodular nonsuppurative panniculitis in association with primary biliary cirrhosis and Hashimoto's thyroiditis. Z Rheumatol. 1996; 55: 122-6.

8. Zhou JY, Xu B, Lopes J, et al. Hashimoto encephalopathy: literature review. Acta Neurol Scand. 2017; 135: 285-90.

9. Shovman O, Gilburd B, Zandman-Goddard G, et al. Pathogenic role and clinical relevance of antineutrophil cytoplasmic antibodies in vasculitides. Curr Rheumatol Rep. 2006; 8: 292-8. 